\section{Spotlight on BACE: The secretases as targets for treatment in Alzheimer disease}

\author{
Colin Dingwall
}

Neurology-CEDD, New Frontiers Science Park, Third Avenue, Harlow CM19 5AW, United Kingdom. Phone: 44-1279-622651; Fax: 44-1279-622555; E-mail: Colin_Dingwall@sbphrd.com

J. Clin. Invest. 108:1243-1246 (2001). DOI:10.1172/JCI200114402.

Alzheimer disease (AD) is a progressive degenerative disease of the brain that is characterized by neocortical atrophy, neuron and synapse loss, and the presence of extracellular senile plaques and intracellular neurofibrillary tangles (NFTs). The primary clinical manifestation of $\mathrm{AD}$ is a profound global dementia that is marked by severe amnesia with additional deficits in language, "executive" functions, attention, and visiospatial and constructional abilities. The neurodegenerative changes occur primarily in the hippocampus and entorhinal cortex and in the association cortices of the frontal, temporal, and parietal lobes. Although the temporal progression of the neuropathological changes of $\mathrm{AD}$ is not fully known, recent studies suggest that the hippocampus and entorhinal cortex are involved in the earliest stage of the disease, and that frontal, temporal, and parietal association cortices develop pathology as the disease progresses. This "spreading" of the pathology from those regions of the brain in which hallmarks of the disease (amyloid plaques, reactive gliosis, NFTs) can be first detected, to other regions of the brain is notable, and while generally accepted as being a genuine feature of the pathology, no explanation for it has yet emerged (1).

As the population ages, the projected number of individuals that will be affected by dementia, and $\mathrm{AD}$ in particular, indicates that a serious public health problem is looming. However, intense research over the past decade has begun to uncover some of the cell and molecular processes leading to neuronal loss with the discovery of possible targets for therapeutic intervention, raising the hope that we may be able at least to halt the progression of the disease $(2,3)$.

The major constituent of senile plaques is the $\beta$-amyloid peptide, which is derived from the amyloid precursor protein (APP) by proteolytic cleavage (3). This peptide is invariably described as a 40 - to 42 -amino acid peptide, although numerous shorter $\mathrm{x}-40$ and $\mathrm{x}-42$ forms are found, particularly in the $\mathrm{AD}$ brain, and many of these peptides have a strong tendency to aggregate (4). By contrast, the intracellular NFTs are fibrillar aggregates of the microtubule-associated protein tau
(5). The vast majority of AD cases are "spontaneous," in that there is no familial history of the disease and hence no known genetic linkage that predisposes an individual to develop AD. However, the rare cases of familial AD have proven to be key in the identification of three genes, APP and presenilins 1 and 2 (PS1 and PS2), that, when mutated, lead to early-onset familial forms of the disease (6). Each of these gene products plays a role in the production of $\beta$-amyloid peptides; APP is the precursor protein from which $\beta$-amyloid peptides are derived by proteolytic cleavage at the $\beta$ and $\gamma$ cleavage sites (Figure 1a). Familial mutations in APP and both presenilins increase the plasma levels of $\beta$-amyloid in patients carrying these mutations and tip the balance toward an increase in the production of $x-42$, the more fibrillogenic species $(7,8)$. Mutations in APP close to the $\beta$ and $\gamma$ cleavage sites (Figure 1b) accelerate amyloid production and increase the proportion of the amyloidogenic $A \beta x-42$ species (3). Presenilin mutations appear to directly affect cleavage site selection and the frequency of cleavage at the $\gamma$-site, and PS1 is absolutely required for $\gamma$-cleavage of APP (3). Early biochemical characterization and inhibitor profiling indicated that both $\beta$ and $\gamma$ cleaving enzymes (secretases) were probably aspartic proteinases and hence represented attractive therapeutic targets (9).

\section{The amyloid hypothesis}

Late in 1999, $\beta$-secretase (BACE, Asp2, Memapsin) was definitively identified as a novel membrane-bound aspartic proteinase, providing the essential tool in the search for specific inhibitors of $\beta$-amyloid production (10). The search for $\gamma$-secretase has proven more difficult and indirect, but the current balance of evidence is consistent with PS1 being a novel transmembrane aspartic proteinase (11). While the amyloid hypothesis would support the idea that these proteases represent attractive therapeutic targets, there has been a significant ongoing debate as to whether the extracellular, amyloid-containing senile plaques, or the intracellular NFTs, occur first in the pathology and hence which may be the causative agent (1-3). In support of the important role of NFTs in neurodegeneration, there is clear evidence that their presence often correlates more closely with dementia. By contrast, abundant amyloid deposits can be detected in the brains of cognitively normal individuals (12).

The proposed pharmacological approach of blocking secretase activity is reminiscent of that taken with atherosclerosis, where rare mutations leading to elevated serum cholesterol levels result in disease, yet therapeutic treatments, for example with statins to inhibit cholesterol biosynthesis, lead to clinical improvement even in patients lacking such mutations. Strong support for the amyloid hypothesis - and hence for the potential 
a

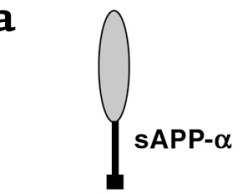

APP

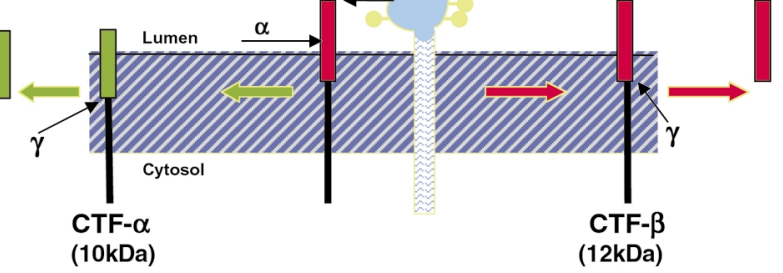

b Secretase cleavage sites in APP

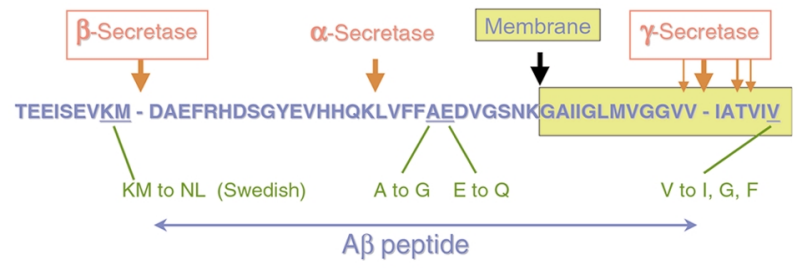

\section{Sequence organization of $\beta$-secretase}

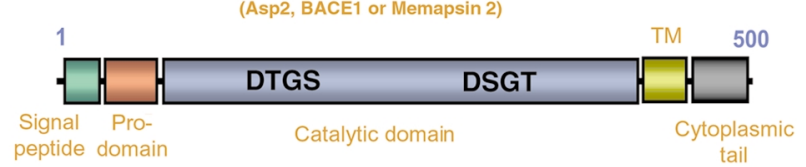

\section{Figure 1}

APP processing pathway and and domain organization of $\beta$-secretase (a) A schematic representation of the sequential cleavages of APP at the $\beta$ - and $\gamma$ - sites to generate $\beta$-amyloid. APP and $\beta$-secretase are both integral membrane proteins with type 1 orientation, $\gamma$-secretase is probably PS 1, a protein with eight TM domains (not shown). Recent data indicates that these three proteins assemble in conjunction with Nicastrin, a protein which interacts with PS1, in cholesterol rich microdomains in cellular membranes. (b) The amyloid domain of APP showing the mutations which are associated with early onset familial forms of $A D$, highlighting those which occur at the secretase cleavage sites. The beginning of the TM domain is shown. (c) Domain organization of $\beta$-secretase. The protein has a cleaved signal peptide, a prodomain and a catalytic domain in which the catalytic aspartic residues are located. The catalytic domain shows the 3 di-sulfide bridges characteristic of the pepsin family of aspartic proteinases and is glycosylated at 4 sites. The cytoplasmic tail contains a di-leucine endosomal retreival signal, cysteine residues which are palmitoylated and phosphorylation sites. The homolog, Asp1 (DRAP, BACE2) shows the same overall structural organization.

value of inhibiting the secretases in an analogous manner - comes from work with both patients and transgenic animals. For instance, one recent study shows a direct correlation between elevated levels of $A \beta$-peptide and cognitive decline in patients (13). In APP transgenic mice, likewise, reduction in amyloid burden through immunization with $\beta$-amyloid peptides restores behavioral deficits seen in the nonimmunized animals (14). Moreover, when transgenic mice expressing a mutant form of human APP that generates high levels of amyloid are crossed with $\beta$-secretase knockout animals, no amyloid can be detected in the double transgenic mice (15-17).

\section{Maturation and function of $\beta$-secretase}

$\beta$-Secretase is a typical aspartic proteinase, 500 amino acids in length with two catalytic aspartic acid residues present in the diagnostic DT/SG sequence motif, $\mathrm{D}^{93} \mathrm{TS}$ and $\mathrm{D}^{289} \mathrm{SG}$. It also has three disulfide bonds characteristic of mammalian enzymes. The crystal structure reveals that the protein has the same general polypeptide fold seen in other members of this family (18) despite an unusual disulfide bonding pattern, which initially led to speculation that the enzyme might reveal novel substrate specificities (19).

In common with other aspartic proteinases, $\beta$-secretase is synthesized as a zymogen with a pro-domain, which is postulated to lie in the active site of the enzyme and thereby maintain it in an inactive state. Cleavage of the pro-domain to generate the mature enzyme occurs on the carboxyterminal side of the sequence $\mathrm{RLPR}^{45}$ to liberate a mature protein starting at $E^{46}$. The RLPR motif is a consensus cleavage site for the proprotein convertase (PC) family of calciumdependent serine proteases, of which seven are known. Two independent studies have exploited the existence of cell lines deficient in the proteinase Furin to demonstrate clearly that this is the predominant PC that catalyzes the removal of the pro-domain of $\beta$-secretase. In one study, LoVo cells, a human colon carcinoma cell line lacking both Furin alleles, were used; in the other, the Chinese hamster ovary (CHO) cell strain RPE.40 was used $(20,21)$. In both cell lines, processing of $\beta$-secretase was dramatically reduced but could be restored by cotransfection of Furin cDNA. Mutation of amino acids in the active site of the transfected $\beta$-secretase cDNA had no effect on this processing, indicating that the enzyme cannot autoactivate $(20,21)$.

Furin itself probably does not offer an attractive target for therapeutic intervention upstream of $\beta$-secretase, since the large number of Furin substrates in the cell and the important biological functions of this class of proteases in vivo suggest that inhibition is likely to have serious deleterious consequences. In addition, there is evidence to indicate that even with the prodomain present, the enzyme is still able to cleave APP (22). So, rather than regulating activity, the predominant role of the prodomain appears to be to ensure efficient folding and trafficking of the protein through the endomembrane system (23).

The approximately 23-amino acid cytoplasmic tail of $\beta$-secretase has a number of sequence elements that suggest multiple functions for this domain. At the extreme carboxyterminus the protein terminates with the sequence DDISLLK. The di-leucine motif is known to be responsible for endosomal and lysosomal targeting from the cell surface (24), while the upstream acidic residues are required for the proper function of this motif (25). Consistent with this notion, deletion of the di-leucine motif from $\beta$-secretase leads to increased levels of protein being resident at the cell surface, consistent with a failure of the deletion mutant to transport to the endosome, indicating that a significant proportion of $\beta$-secretase cycles between the plasma membrane and the endosome. In this cytoplasmic domain there are also three cysteine residues that have been shown to be 
palmitoylated and may help anchor the protein to the membrane. Indeed, although changing each of these residues to alanine to prevent palmitoylation does not lead to major changes in subcellular distribution of the protein, it significantly enhances the shedding of $\beta$-secretase into the medium (22). Shedding, which is the release of the extracellular domain of proteins from their membrane anchor, is often catalyzed by members of the ADAM (a disintegrin and a metalloproteinase) family. The release of the soluble domain of APP by cleavage at the $\alpha$-site (Figure 1 ) is catalyzed by ADAM10 and occurs predominantly at the cell surface $(26,27)$, while the cleavage of $\beta$-secretase from its membrane anchor by a currently unknown proteinase appears to be predominantly an intracellular event (19). The role of shed $\beta$-secretase is also unknown, but it seems unlikely that it is active as a protease, given the neutral $\mathrm{pH}$ of the extracellular environment and the acidic $\mathrm{pH}$ optimum required for efficient substrate cleavage.

\section{$\gamma$-Secretase as a therapeutic target}

The cleavage of APP by $\gamma$-secretase is unusual in that it occurs within the transmembrane domain of APP. This event is not unprecedented, since the sterol response element binding protein is cleaved within its transmembrane domain in response to falling cellular sterol levels (28). The APP cleavage event is strictly dependent upon the presence of PS1, as is the intramembrane cleavage of Notch (29). PS1 is a polytopic protein that has eight transmembrane domains. Cleavage of PS1 between transmembrane domains 6 and 7 to generate two associated fragments appears to be necessary for its ability to promote APP cleavage at the $\gamma$-site. As $\beta$-amyloid can terminate at two different sites to give $\mathrm{x}-40$ or $\mathrm{x}-42$, early studies concluded that there were at least two separate enzymes catalyzing $\gamma$-cleavage, a pepstatin-sensitive activity and a pepstatin-insensitive activity (30-32). However, detailed studies of structurally related aspartic proteinase inhibitors concluded that there is probably a single $\gamma$-secretase, as a clear linear relationship emerged between the rank order potency for inhibition of $x-40$ production and $x-42$ production (33). A detailed mutational analysis of PS1 indicated that mutation of individual aspartic acid residues in transmembrane helices 6 and 7 blocked $\gamma$-secretase cleavage of APP and autocatalytic processing in the loop region between these two transmembrane helices $(34,35)$. The conclusion at this point was that either PS1 is $\gamma$-secretase or it is an essential di-aspartyl cofactor for $\gamma$-secretase. Perhaps the most striking biochemical result came from the use of radiolabeled photoactivatable transition state analog inhibitors; the only proteins to be labeled in a membrane preparation enriched in $\gamma$-secretase activity were the PS1 cleavage fragments (36, 37). Detergent solubilization and immunoprecipitation of PS1 from such a membrane preparation revealed clear coprecipitation with $\gamma$-secretase activity (38). While ultimate proof will consist of reconstituting $\gamma$-secretase activity from recombinant proteins, this series of experiments appears to many to be definitive evidence that PS1 is $\gamma$-secretase.
Already a number of potent and selective $\gamma$-secretase inhibitors have been described by a number of groups and have been shown to block both $\mathrm{x}-40$ and $\mathrm{x}-42$ production in cells (39-41) and in APP transgenic mice, reducing $\beta$-amyloid burden appreciably within a few hours of oral dosing (42). These inhibitors also block PS1 cleavage but with a lower potency, a finding that is explained by suggesting that here the inhibitor is competing with a different substrate sequence for which the enzyme is likely to have a different affinity (43). One potential problem needs to be addressed: several studies of the dose-response profiles of these $\gamma$-secretase inhibitors show that they stimulate $A \beta x-42$ production at low concentrations, while higher concentrations inhibit $A \beta x-42$ production (39-41). This event can be reconstituted in a purified membrane preparation, suggesting that the effect seen in cells is probably not due to an effect of the inhibitor on $A \beta$ trafficking through the cell or release from an intracellular pool (44). One possible explanation is that $\beta$-amyloid is rapidly degraded by another proteinase that is still more sensitive to the inhibitor than is $\gamma$-secretase, so that the inhibition of the unknown proteinase leads to the accumulation of $A \beta x-42$. As concentrations rise, $\gamma$-secretase is inhibited, blocking the production of $\beta$-amyloid upstream of the degradative proteinase. Clearly this stimulatory activity cannot be present in any inhibitor that is to be considered for clinical application.

\section{Other strategies for therapeutic blockade of APP processing}

A number of in vivo and in vitro studies have shown that $\beta$-amyloid production is sensitive to cholesterol levels, and, while some of the published results are contradictory, the bulk of them consistently indicate that high cholesterol ester levels correlate with increased amyloid production both in cells and in transgenic animals expressing human APP. Hence the $\beta$ - and $\gamma$-secretase activities may be positively regulated by cholesterol (45). In contrast, the $\alpha$-secretase activity appears to be negatively regulated by cellular cholesterol (46). Consistent with these in vivo and in vitro data, epidemiological studies show a reduced prevalence of $\mathrm{AD}$ in patients treated with inhibitors of cholesterol biosynthesis (47). Cellular cholesterol may act in part by partitioning the proteinases into distinct lipid microenvironments within the plasma membrane. In the membrane, cholesterol-enriched microdomains or "rafts" are zones in which proteins involved in signal transduction, protein trafficking, and proteolytic processing accumulate (48). Rafts can be partially purified because of their relative insolubility in detergent at low temperature and their different buoyant density compared with the bulk of the cellular membrane (48). In this way, APP, $\beta$-amyloid, and the putative $\gamma$-secretase PS1 have all been found in lipid rafts. Recently, $\beta$-secretase protein and activity have been found in a light membrane raft fraction that also contained other components of the amyloidogenic pathway, and depletion of cellular cholesterol significantly reduced the amount of $\beta$-secretase in this fraction (49). These observations suggest that the cholesterol-dependent 
partitioning of $\beta$-secretase into lipid rafts to promote its association with APP and PS1 to generate a proteolytically active assembly may underlie the cholesterol sensitivity of $\beta$-amyloid production.

\section{Prospects for clinical control of AD}

Whether prevention of amyloid deposition, achieved by any of the strategies discussed above, would be therapeutic depends critically upon whether amyloid plaques, and particularly soluble fibrillar amyloid, are neurotoxic. The most detailed studies suggest that a discrete form of soluble protofibril is the toxic species. Also consistent is the finding that cognitive decline in humans and behavioral deficits in APP transgenic animals correlate more closely with soluble $\beta$-amyloid levels than with plaque deposition $(13,14)$. If so, the secretases remain the most promising targets available for the treatment of AD.

\section{Acknowledgments}

I would like to thank David Howlett and Ishrut Hussain for helpful discussions and comments on the manuscript. In the interests of space, key review articles are cited within which references to the primary literature may be found.

1. Salmon, P.D. 1997. Neuropsychological features of Alzheimer's disease. In Pharmacological treatment of Azheimer's disease: molecular and neurobiological foundations. J.D. Brioni and M.W. Decker, editors. Wiley-Liss Inc. New York, New York, USA. 129-147.

2. Chapman, P.F., Falinska, A.M., Knevett, S.G., and Ramsay, M.F. 2001 Genes, models and Alzheimer's disease. Trends Genet. 17:254-261.

3. Selkoe, D.J. 2001. Alzheimer's disease: genes, proteins and therapy. Physiol. Rev. 81:741-766.

4. Haass, C., and Steiner, H. 2001. Protofibrils, the unifying toxic molecule of neurodegenerative disorders? Nat. Neurosci. 4:859-860.

5. Goedert, M., et al. 1989. Multiple isoforms of human microtubule-associated protein tau: sequences and localization in neurofibrillary tangles of Alzheimer's disease. Neuron. 3:519-526.

6. Haass, C. 1997. Presenilins: genes for life and death. Neuron. 18:687-690.

7. Schuener, D., et al. 1996. Secreted amyloid $\beta$-protein similar to that in the senile plaques of Alzheimer's disease is increased in vivo by the presenilin 1 and 2 and APP mutations linked to familial Alzheimer's disease. Nat. Med. 2:864-870.

8. Borchelt, D.R., et al. 1996. Familial Alzheimer's disease-linked presenin 1 variants elevate $A \beta \quad 1-42 / 1-40$ ratio in vitro and in vivo. Neuron. 17:1005-1013.

9. Wolfe, M.S., et al. 1999. Peptidomimetic probes and molecular modeling sugest that Alzheimer's $\gamma$-secretase is an intramembrane-cleaving aspartyl protease. Biochemistry. 38:4720-4727.

10. De Strooper, B., and Konig, G. 1999. A firm base for drug development. Nature. 402:471-472.

11. Vassar, R., and Citron, M. 2000. A $\beta$-generating enzymes: recent advances in $\beta$ - and $\gamma$-secretase research. Neuron. 27:419-422.

12. Spillantini, M.G., and Goedert, M. 1998. Tau pathology in neurodegenerative diseases. Trends Neurosci. 21:428-433.

13. Naslund, J., et al. 2000. Correlation between elevated levels of amyloid $\beta$ peptide in the brain and cognitive decline. JAMA. 12:1571-1577.

14. Younkin, S. 2001. Amyloid $\beta$ generation: reduced plaques and improved cognition. Nat. Med. 1:8-19.

15. Luo, Y., et al. 2001. Mice deficient in BACE1, the Alzheimer's $\beta$-secretase, have normal phenotype and abolished $\beta$-amyloid generation. Nat. Newrosci. 4:231-232.

16. Cai, H., et al. 2001. BACE is the major $\beta$-secretase for generation of $A \beta$ peptides by neurons. Nat. Neurosci. 4:233-234.

17. Roberds, S.L., et al. 2001. BACE knockout mice are healthy despite lacking the primary $\beta$-secretase activity in brain: implications for Alzheimer's disease therapeutics. Hum. Mol. Genet. 10:1317-1324.

18. Hong, L., et al. 2000. Structure of the protease domain of Memapsin 2 ( $\beta$ secretase) complexed with inhibitor. Science. 290:150-153.

19. Haniu, M., et al. 2000. Characterization of Alzheimer's $\beta$-secretase protein BACE. J. Biol. Chem. 175:21099-21106.
20. Bennett, B.D., et al. 2000. A furin-like convertase mediates the propeptide cleavage of BACE, the Alzheimer's $\beta$-secretase. J. Biol. Chem. 275:37712-37717.

21. Creemers, J.M.W., et al. 2000. Processing of $\beta$-secretase by furin and other members of the proprotein convertase family. J. Biol. Chem. 276:4211-4217.

22. Benjannet, S., et al. 2001. Post-translational processing of $\beta$-secretase ( $\beta$ amyloid-converting enzyme) and its ectodomain shedding. J. Biol. Chem. 276:10879-10887.

23. Shi, X.-P., et al. 2000. The pro domain of $\beta$-secretase does not confer strict zymogen-like properties but does assist proper folding of the protease domain. J. Biol. Chem. 276:10366-10373.

24. Sandoval, I.V., and Baake, O. 1994. Targeting of membrane proteins to endosomes and lysosomes. Trends Cell Biol. 4:292-297.

25. Pond, L., et al. 1995. A role for acidic residues in di-leucine motif-based targeting to the endocytic pathway. J. Biol. Chem. 270:19989-19997.

26. Parvathy, S., et al. 1999. Cleavage of Alzheimer's amyloid precursor protein by $\alpha$-secretase occurs at the surface of neuronal cells. Biochemistry. 38:9728-9734.

27. Lammich, S., et al. 1999. Constitutive and regulated $\alpha$-secretase cleavage of Alzheimer's amyloid precursor protein by a disintegrin metalloprotease. Proc. Natl. Acad. Sci. USA. 96:3922-3927.

28. Brown, M.S., and Goldstein, J.L. 1997. The SREBP pathway: regulation of cholesterol metabolism by proteolysis of a membrane-bound transcription factor. Cell. 89:331-340.

29. Hardy, J., and Isreal, A. 1999. In search of $\gamma$-secretase. Nature. 398:466-467.

30. Murphy, M.P., et al. 1999. $\gamma$-Secretase, evidence for multiple proteolytic activities and influence of membrane positioning of substrate on generation of amyloid $\beta$ peptides of varying length. J. Biol. Chem. 274:11914-11923.

31. Citron, M., et al. 1996. Evidence that the 42 and 40 -amino acid forms of amyloid $\beta$ protein are generated from the $\beta$-amyloid precursor protein by different activities. Proc. Natl. Acad. Sci. USA. 93:13170-13175.

32. Kalfki, H.-W., et al. 1996. The carboxytermini of $\beta$-amyloid peptides 1 40 and $1-42$ are generated by distinct $\gamma$-secretase activities. J. Biol. Chem. 271:28655-28659.

33. Durkin, J.T., et al. 1999. Rank-order potencies for inhibition of the secretion of $A \beta 40$ and $A \beta 42$ suggest that both are generated by a single $\gamma$-secretase. J. Biol. Chem. 274:20499-20504.

34. Kimberley, W.T., et al. 2000. The transmembrane aspartates in presenilin 1 and 2 are obligatory for $\gamma$-secretase activiy and amyloid $\beta$-protein generation. J. Biol. Chem. 275:3173-3178.

35. Wolfe, S.M., et al. 2000. Two transmembrane aspartates in presenilin-1 required for presenilin endoproteolysis and $\gamma$-secretase activity. Nature. 398:513-517.

36. Esler, W.P., et al. 2000. Transition-state analogue inhibitors of $\gamma$-secretase bind directly to presenilin-1. Nat. Cell Biol. 2:428-434.

37. Li, Y.-M., et al. 2000. Photoactivated $\gamma$-secretase inhibitors directed to the active site covalently label presenilin 1. Nature. 405:689-693.

38. Li, Y.-M., et al. 2000. Presenilin 1 is linked with $\gamma$-secretase activity in the detergent solubilized state. Proc. Natl. Acad. Sci. USA. 97:6138-6143.

39. Wolfe, M.S., et al. 1998. A substrate-based difluoro ketone selectively inhibits Alzheimer's $\gamma$-secretase activity. J. Med. Chem. 41:6-9.

40. Shearman, M.S., et al. 2000. L-685,458, an aspartyl protease transition state mimic, is a potent inhibitor of amyloid $\beta$-protein precursor $\gamma$-secretase activity. Biochemistry. 39:8698-8704.

41. Rishton, G.M., et al. 2000. Fenchylamine sulfonamide inhibitors of amyloid $\beta$-peptide production by the $\gamma$-secretase proteolytic pathway: potential small molecule therapeutic agents for the treatment of Alzheimer's disease. J. Med. Chem. 43:2297-2299.

42. Dovey, H.F., et al. 2001. Functional gamma-secretase inhibitors reduce beta-amyloid peptide levels in brain. J. Neurochem. 76:173-181.

43. Beher, D., et al. 2001. Pharmacological knock-down of the presenilin 1 heterodimer by a novel $\gamma$-secretase inhibitor: implications for presenilin biology. J. Biol. Chem. In press.

44. Zhang, L., et al. 2001. Biochemical characterization of the $\gamma$-secretase activity that produces $\beta$-amyloid peptides. Biochemistry. 40:5049-5055.

45. Golde, T.E., and Eckman, C.B. 2001. Cholesterol modulation as an emerging strategy for the treatment of Alzheimer's disease. Drug Discov. Today. 6:1049-1055.

46. Bodovitz, S., and Klein, W.L. 1996. Cholesterol modulates $\alpha$-secretase cleavage of amyloid precursor protein. J. Biol. Chem. 271:4435-4440.

47. Wolozin, B., et al. 2000. Decreased prevalence of Alzheimer disease associated with 3-hydroxy-3-methlyglutaryl coenzyme A reductase inhibitors. Arch. Neurol. 57:1439-1443.

48. Simons, K., and Toomre, D. 2000. Lipid rafts and signal transduction. Nat. Rev. Mol. Cell Biol. 1:31-39.

49. Riddell, D., et al. 2001. Compartmentalization of $\beta$-secretase (Asp2) into low-buoyant density non-cavoelar lipid rafts. Curr. Biol. 11:1288-1293. 